\title{
¿Contribuyen las Redes de Capital Social a la Innovación en los centros educativos?: Análisis en los Centros de Enseñanza de Bachillerato de la Comunidad Valenciana
}

\author{
Do Social Capital Networks contribute to Innovation in Educational \\ Centers: Analysis in High Schools from the Valencian Community
}

\author{
Rubén J. Cuñat Giménez ${ }^{1}$ \\ ${ }^{1}$ Universitat de València. ruben.cunat@uv.es
}

Recibido: $30 / 5 / 2020$

Aceptado: 23/10/2020

Copyright (C)

Facultad de CC. de la Educación y Deporte. Universidad de Vigo

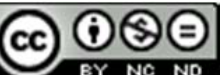

Dirección de contacto: Rubén Cuñat Giménez

Departamento de Economía Aplicada Facultat d'Economia

Campus de Tarongers

Avda. dels Tarongers, $\mathrm{s} / \mathrm{n}$

46022 Valencia

\begin{abstract}
Resumen
Este trabajo pretende aportar datos sobre la efectividad de las redes de capital social en los centros de enseñanza de bachillerato de la Comunidad Valenciana, aportando datos sobre las dimensiones que participan en las redes y su impacto sobre la innovación educativa. Para ello, se ha obtenido información de 71 centros repartidos proporcionalmente por toda la Comunidad Valenciana, con el objetivo de identificar la contribución de cada una de las dimensiones relacionadas con redes sociales sobre la innovación educativa, observando las diferencias entre los centros públicos y privados y la efectividad de estas redes.
\end{abstract}

Se puede constatar que existen diferencias en el grado de innovación entre los centros públicos y privados de bachillerato, que solo las normas y valores tienen incidencia sobre la innovación y que el tamaño del centro no repercute directamente en el nivel de innovación de éste.

Finalmente, el trabajo muestra que el tiempo que se dedica a las relaciones de red, su intensidad y frecuencia no tiene los efectos que se podría esperar, sino que es la dimensión confianza y reputación la que ejerce un mayor impacto para favorecer la transmisión de conocimiento y por lo tanto la generación de capital social.

\section{Palabras clave}

Innovación Educativa, Bachillerato, Educación, Capital Social, Redes Sociales

\section{Abstract}

This paper aims to provide data on the effectiveness of social capital networks in the high school of the Valencian Community, providing data on the dimensions involved in the networks and their impact on educational innovation. For this purpose, we have collected information from 71 centers distributed proportionally throughout the Valencian Community to identify the contribution of each of the dimensions related to social networks on educational innovation, observing the differences between 
public and private centers and the effectiveness of these networks. It can be seen that there are differences in the degree of innovation between public and private high school establishments, that only the rules and values have an impact on innovation and the size of the center does not directly affect the innovation level of this one.

Finally, the work shows that the time spent on network relationships, their intensity and frequency do not have the effects we could expect, but it is the confidence and reputation dimension that has the greatest impact to promote the transmission of knowledge and therefore the generation of social capital.

\section{Key Words}

Educational Innovations, High Schools, Education, Social Capital, Social Networks

\section{INTRODUCCIÓN}

Actualmente no podemos entender un mundo con personas o instituciones que actúan de forma aislada, ni tampoco actores que no son capaces de utilizar el conocimiento compartido para conseguir mejorar sus niveles de eficiencia, eficacia y mejora social. Dentro de este contexto, el capital social se convierte en un vehículo que impulsa el conocimiento compartido perteneciente a distintas organizaciones o grupos (Chow y Chan, 2008), siendo las redes sociales instrumentos que nacen con el propósito de incrementar ese conocimiento (Almuiñas y Galarza, 2016).

La Innovación como término relacionado con la capacidad para modificar, cambiar o perfeccionar alguna cosa incorporando algo nuevo o novedoso dentro de una realidad existente, constituye un elemento fundamental para transformar la sociedad. Es por esta razón por lo que en el ámbito educativo la innovación cobra especial relevancia. La innovación no es un concepto nuevo, sino un concepto que ha ido adaptándose a los cambios que se han producido en la sociedad (Casillas, Cabezas y Navarro, 2013). La innovación educativa se sustenta en cuatro pilares básicos: los procesos, el conocimiento, las personas y las tecnologías, siendo sus principales objetivos promover actitudes positivas hacia el cambio y crear espacios que permitan identificar, valorar, sistematizar, normalizar, aplicar y difundir experiencias novedosas para la solución de problemas educativos.

El proceso de enseñanza-aprendizaje es efectivo cuando todos los agentes que participan lo perciben abierto a la mejora continua e integrado en un proyecto curricular de carácter institucional, siendo necesaria la interacción creativa y colaborativa para alcanzar los objetivos propuestos. Pero esta interacción no es suficiente para la transmisión y utilización efectiva del conocimiento, pues la mera interacción no conlleva la utilización de éste para la mejora y tampoco presupone la generación de capital social. Es por tanto necesario que analicemos los efectos que tienen las conexiones de redes en el mundo educativo para generar cambios y al mismo tiempo buscar los motivos, si es el caso, por el que estas interacciones no tienen el impacto que deberían tener sobre la innovación educativa. 


\section{CAPITAL SOCIAL Y REDES: DIMENSIONES DE ANÁLISIS}

El capital social se define como los recursos incrustados en una red social y que pueden adquirirse o movilizarse a través de conexiones dentro de una red (Lin, 2001). De las múltiples aproximaciones que podemos encontrar sobre el tema en la literatura, las redes y la confianza son los dos principales componentes de este concepto. Mientras la primera se materializa en el marco de las relaciones interpersonales, la segunda permanece dentro de la mente de cada persona (valores), convirtiéndose en capital social cuando se comparte (Azqueta, Gavaldón y Margalef, 2007).

Las redes sociales permiten a las organizaciones tener acceso a recursos que actuando de forma individualizada serían difíciles de conseguir, tales como bienes, servicios e Innovación. Tal como indican Elfring y Hulsink (2003), las redes constituyen uno de los capitales más importantes que una organización puede poseer, dado que son capaces de proporcionar poder, información, conocimiento y capital, así como otras redes. Algunos autores, como Venkataraman y Van de Ven (1998) indican que la supervivencia y crecimiento de una organización depende de su habilidad para mantener y extender sus redes, pues éstas generan capacidades de aprendizaje, incrementan la habilidad para innovar, introducir nuevos productos o servicios y descubrir nuevas oportunidades.

Para Hung (2006) las redes sociales tienen una doble naturaleza: interpersonal (formadas por lazos de tipo familiar, amigos, colegas, contactos de negocios y afiliaciones a asociaciones profesionales), que son usadas por los individuos que componen una organización para relacionarse con otros individuos o grupos, con el objetivo de buscar acceso a recursos y fuentes de información relevantes; y la interorganizacional (relacionada con el mercado), que permite mejorar la ventaja competitiva de la organización a través de la minimización de los costes de transacción, manteniendo la flexibilidad y la independencia (Herrera, 2009). Para Casson y Giusta (2007) el crecimiento de una organización se logra fundamentalmente a través del fortalecimiento de las redes interorganizacionales, aunque el mantenimiento de las personales es fundamental, debido a que las redes interorganizacionales se mantienen en la práctica a través de las comunicaciones interpersonales entre los representantes de las organizaciones; de forma que muchas veces un cambio en las personas puede influir en las relaciones entre las organizaciones si el cambio no ha sido gestionado convenientemente por ésta.

Desde la perspectiva del capital social se pueden encontrar dos dimensiones de las redes sociales, por una parte, la dimensión relacional, que hace referencia a la fortaleza de la relación entre individuos u organizaciones y la riqueza de la información intercambiada; y por otra la dimensión estructural, que está relacionada con la densidad, siendo el principal origen de la información y conocimiento que adquiere la organización (información procedente de organizaciones, personas o instituciones con las que mantiene contactos frecuentes o de relaciones esporádicas con otros agentes) (Molina, 2005).

La fortaleza de los lazos entre organizaciones está formada por una combinación de la cantidad de tiempo, intensidad en los contactos y la frecuencia de los contactos, es decir, el número de veces que una organización mantiene contacto con otras durante una unidad de tiempo (Granovetter, 1973), mientras que la riqueza se relaciona con la 
calidad de la información y los conocimientos recibidos por parte de la organización. Por otra parte, la dimensión estructural mide el grado en el que la red está interconectada, y el peso que tiene ésta para que una organización consiga la información y los recursos que necesita a través de ella (Burt, 1992).

A estas dos dimensiones, algunos autores como Tsai y Ghoshal (1998) incorporan una nueva dimensión relacionada con normas y valores y que consideran como un factor adicional que contribuye a generar valor en forma de innovación. La confianza actúa como mecanismo de control sobre los intercambios entre los actores pertenecientes a la red y permite intercambiar información sin el riesgo de comportamientos oportunistas por parte de los participantes.

Las redes de naturaleza interorganizacional (relacionadas con el entorno) son las que más impacto tienen sobre la innovación; sin embargo, para que una red sea efectiva y eficiente deben estar presentes un conjunto de acciones, actitudes o factores básicos, tales como la confianza, el compromiso, la comunicación, la coordinación y la participación (Cabaleiro, 2001).

Estas acciones dependen fundamentalmente del capital humano, máxime si consideramos que los centros de enseñanza son organizaciones personalistas, intensivas en mano de obra y por tanto dependientes de las actitudes que sus partícipes desarrollan respecto a sus perspectivas de futuro.

El estudio de las redes de capital social en la literatura especializada relacionado con la educación ha sido escasamente tratado, como se desprende del estudio realizado por López, Civís y Díaz-Gibson (2018) que realizaron una búsqueda exhaustiva sobre el tema y finalmente solo encontraron 14 estudios específicos entre los años 2000 y 2015 a nivel internacional; años donde hubo un número importante de publicaciones dentro del ámbito educativo en relación a las redes sociales y al capital social (Carolan, 2013). A nivel nacional no se han encontrado referencias que tratan este tema en particular.

\section{FACTORES QUE INCIDEN EN LA INNOVACIÓN EDUCATIVA EN UN CENTRO DE ENSEÑANZA}

La innovación es un concepto muy amplio que incluye numerosas definiciones y categorías, no obstante, es importante no confundir innovar con inventar, puesto que la innovación se refiere a aprovechar todo lo que tenemos a nuestro alcance y adaptarlo a nuestras necesidades. Se trata de institucionalizar las mejores prácticas y permitir asumir compromisos que garanticen su permanencia en el tiempo.

Por otra parte, se sabe que dada su estructura normativa, las escuelas generalmente han sido instituciones poco innovadoras, sin confundir con su dinamismo en la generación de proyectos innovadores desde el aula a partir de las inquietudes de su propio profesorado. El profesor Francesc Llorens (2011, pág. 315) define innovación en el contexto de la formación como "la producción y difusión de prácticas que supongan ventajas, mejoras $\mathrm{u}$ oportunidades con respecto al modo en que se concibieron y desempeñaron en el pasado; y ello en cualquier contexto imaginable". Considera que, aunque detrás del profesorado están las instituciones y son los centros los que conciben modelos y desarrollan estrategias, sin la actitud y la predisposición de los profesores y profesoras en un centro éste no puede conseguir el éxito en sus metas organizativas hacia la innovación. 
La innovación educativa se origina y desarrolla en diferentes contextos (Rosales, 2012):

- Aula. Corresponde a todas las actividades didácticas que se realizan en el interior de ésta con referencia a cambios innovadores, incluyendo la utilización de las TIC para impulsar cambios pedagógicos (González y De Pablos, 2015). La incorporación de las TIC en el aula genera procesos de innovación pedagógica que influyen en el papel del docente y estudiante, además de cambios en los espacios físicos, utilización de recursos, etc. (Parra, Gómez y Pintor, 2015).

- Centro Escolar. Responde a la voluntad del centro sobre innovar o permanecer en su situación actual condicionado por su dirección y por el grado de autonomía o dependencia respecto a las normas administrativas y legales. Las prácticas de renovación pedagógica representan una manera diferente de abordar los procesos educativos promoviendo la innovación constante con el fin de mejorar la respuesta de la institución a los retos de la sociedad (Pericacho, 2014).

- Administración Educativa. Ésta, con su actitud de centralización o descentralización en la toma de decisiones por parte de los centros, puede condicionar la capacidad de éstos para innovar.

- Comunidad. Que incorpora una doble vertiente: por una parte, la adaptación del centro a las características de la comunidad en la que se ubica, atendiendo tanto a los aspectos sociales del presente y del futuro, y por otra parte la vinculación de la escuela en su entorno social. La colaboración y participación de la administración educativa y las familias se constituye como aspecto clave en el proceso de innovación.

En opinión del profesor Pere Marquès (2005), los cambios en los centros docentes deben incluir varios escenarios relacionados con los Recursos Materiales, los Recursos Humanos y los Recursos Funcionales.

Por lo que respecta a los Recursos Materiales, estos incluyen:

- Las Infraestructuras y los Modelos Tecnológicos de uso de las TIC. Siendo importante que los centros dispongan de recursos tecnológicos correctamente ubicados e instalados, con los programas y recursos digitales necesarios, así como un apropiado sistema de mantenimiento en cada centro. Los ordenadores, internet y las pizarras digitales interactivas suelen ser las tecnologías más utilizadas en los centros (Colás, De Pablos y Ballesta, 2018)

- Materiales de Apoyo a la Educación. Incluye materiales didácticos, tanto materiales convencionales como libros, juegos, videos, etc., como materiales multimedia y del ciberespacio que pueden aportar un tratamiento personalizado al alumnado, además de plataformas de contenidos en red.

Sin embargo, las infraestructuras y los materiales de apoyo no son suficientes para desarrollar procesos de innovación sin la implicación de las personas y la creación de un clima de aceptación de las tecnologías que permitan mejorar los procesos de aprendizaje. En este sentido hay que prestar atención a los siguientes participantes en el proceso de innovación docente:

- El Profesorado. Constituye el eje central de todo proceso de Innovación, en cuanto que éste influye en el éxito de los estudiantes a partir de su propia formación, actitud y motivación. La preparación del profesorado es fundamental para 
garantizar la adecuada introducción de prácticas innovadoras en el aula, seguida de una motivación específica hacia la enseñanza (Muñoz-Fernández, RodríguezGutiérrez y Luque-Vílchez, 2019).

- El Equipo Directivo. En un entorno tan diverso y cambiante como el actual, la preparación del equipo directivo debe acompañarse de planes formativos que incluyan las nuevas competencias que hoy en día exige la función directiva. Además, la gestión educativa debe atender de manera prioritaria el liderazgo de la dirección de la institución.

- El Personal no docente. La labor del personal no docente en el centro, sobre todo en materia de integración de las TIC, es fundamental para garantizar aspectos logísticos básicos de funcionamiento.

- El Alumnado. En el entorno actual, el papel del estudiante debe cambiar. Es necesario incrementar su grado de motivación, superando una estructura de trabajo de tipo individualista hacia una estructura de trabajo de tipo cooperativo.

- Las Familias. La familia es el agente educativo que mayor influencia tiene sobre todo ser humano, compartiendo su labor educativa con el entorno y con las instituciones educativas. La institución y los progenitores permiten intercambiar informaciones y formas de pensar para el desarrollo del aprendizaje del alumnado.

- El Entorno Social. Las entidades educativas, culturales y deportivas del entorno pueden contribuir al desarrollo de los estudiantes, además de conseguir una adecuada complicidad que implique un creciente protagonismo de la educación.

Y, por último, respecto a los Recursos Funcionales, es importante que el centro docente refleje el compromiso con la innovación a través del Proyecto Educativo del Centro, en el que se deben dar a conocer los valores que se quieren transmitir, las metas humanas, culturales y sociales, así como las pautas para el desarrollo cognitivo/emotivo del alumnado, diseñando un modelo educativo que identifique al centro (González y De Pablos, 2015). Las actividades deben favorecer el desarrollo de procesos de aprendizaje que incentiven y contribuyan a la reflexión y el aprendizaje autónomo del alumnado (García-Valcárcel, Hernández y Recamán, 2012).

\section{MÉTODO}

\subsection{Objetivos e Hipótesis}

A partir del marco teórico definido en este trabajo, se plantean cuatro hipótesis (Figura 1). En un primer análisis, se estudia cmo influyen las distintas variables de redes de capital social en la generación de innovación educativa (hipótesis H1). En este sentido, teniendo en cuenta las referencias comentadas en este trabajo, cuanto más densa y menos dispersa es una red teóricamente mayor es la calidad de la información y recursos que se obtienen y por tanto la organización debería incrementar su capacidad de innovar. Para contrastar esta hipótesis, se lleva a cabo un análisis empírico a partir de un modelo de regresión lineal múltiple. Previamente se realiza un análisis factorial de componentes principales para evitar posibles problemas de colinealidad y obtener una agregación y normalización de las variables de capital social.

Finalmente, dentro de este primer estudio se analiza si el tamaño del centro educativo incide en el grado de innovación de este (hipótesis H2). 
En segundo lugar (hipótesis H3 y H4), se trata, por una parte, de conocer si los centros educativos públicos y privados difieren en la naturaleza de conexión con redes de capital social. Es decir, se analiza si ambas muestras tienen importantes disparidades en las dimensiones teóricas definidas en este trabajo con referencia a las redes sociales.

Para ello, se realiza un análisis empírico utilizando como modelo estadístico una prueba $t$ de Student para la igualdad de medias, que permite conocer si hay diferencias significativas en las medias de las variables que conforman las redes de capital social. A continuación, se estudia si el tipo de centro (público o privado) incide en el grado de innovación del mismo a través de un contraste de medias respecto a esta variable.

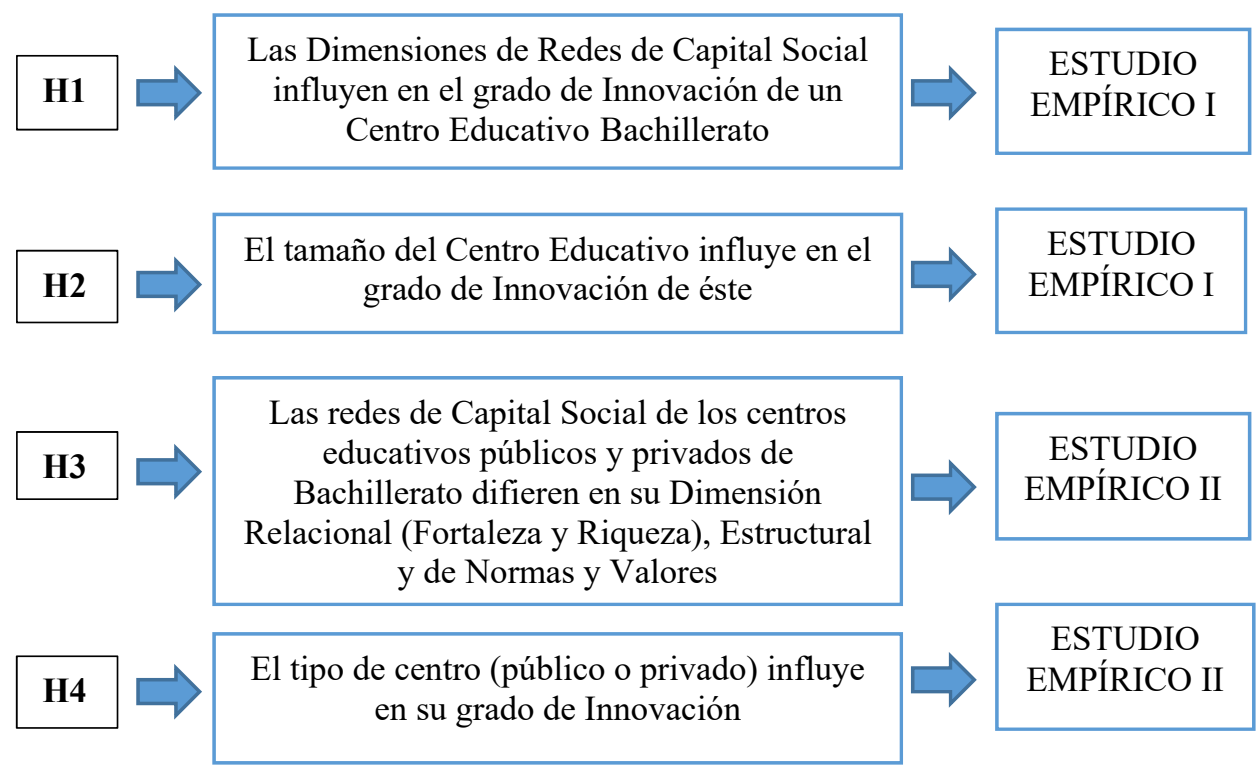

Figura 1. Hipótesis de Estudio

\subsection{Procedimiento y muestra}

Para la confección de la muestra de estudio se accedió a la base de datos pública que dispone la Consellería d'Educació, Investigació, Cultura i Esport de la Generalitat Valenciana sobre centros de Bachillerato en la Comunidad Valenciana. Se envió un cuestionario vía e-mail en formato electrónico a todos los centros educativos que imparten Bachillerato registrados en esta base de datos durante el mes de octubre del 2018 (331 centros públicos y 140 privados), obteniendo 71 respuestas de noviembre a enero del 2019 (55 centros públicos y 16 privados).

El cuestionario fue diseñado atendiendo a las proposiciones teóricas de nuestra investigación e incluía preguntas de valoración, utilizando una escala tipo Likert 1-5 (1 total desacuerdo y 5 total acuerdo) donde se presentaban preguntas relacionadas con las dimensiones de estudio. Las variables utilizadas para medir el capital social no eran directamente observables, por lo que se hicieron operativas a través de escalas de medida. Esto implicaba formular preguntas relacionadas con las dimensiones descritas en este estudio. En este sentido, se formularon 12 ítems relacionados con las redes sociales y 37 ítems relacionados con la innovación.

Los ítems relacionados con las redes sociales, así como los bloques en que fueron agrupados para facilitar la respuesta y comprensión, se pueden ver en la Tabla 1. 


\begin{tabular}{|c|c|}
\hline $\begin{array}{l}\text { BLOQUE DE } \\
\text { ANÁLISIS }\end{array}$ & Ítems \\
\hline \multirow{3}{*}{ BLOQUE I } & 1.1 Cuando se reúne con otros centros educativos se comparte información \\
\hline & $\begin{array}{l}\text { 1.2 Conoce a todos/as los representantes de los diferentes centros educativos de } \\
\text { su comarca }\end{array}$ \\
\hline & 1.3 Mantiene relaciones con otros centros educativos \\
\hline \multirow{3}{*}{ BLOQUE II } & 2.1 Comparte ciertos objetivos comunes con otros centros educativos \\
\hline & $\begin{array}{l}\text { 2.2 Hay profesores/as o personal del centro que han trabajado en otros centros } \\
\text { dentro de la zona en años anteriores }\end{array}$ \\
\hline & $\begin{array}{l}\text { 2.3 Participa junto a otros centros o proveedores en eventos sociales, } \\
\text { celebraciones, etc. localizadas en su área }\end{array}$ \\
\hline \multirow{3}{*}{ BLOQUE III } & $\begin{array}{l}\text { 3.1 La información, conocimiento, consejos que recibe su centro de otros } \\
\text { organismos y/o instituciones le ayudan a la resolución de problemas }\end{array}$ \\
\hline & $\begin{array}{l}\text { 3.2 La transferencia de información entre los centros beneficia a todos los } \\
\text { colegios incrementando sus posibilidades de conseguir mejores resultados }\end{array}$ \\
\hline & $\begin{array}{l}\text { 3.3 Las relaciones que mantiene con organizaciones externas incluyen } \\
\text { información con veracidad y significado que va más allá de lo aparente o } \\
\text { explícito }\end{array}$ \\
\hline \multirow{3}{*}{ BLOQUE IV } & $\begin{array}{l}\text { 4.1 En general considera que en sus relaciones con familias, otros centros de } \\
\text { enseñanza, proveedores, etc. existe un total grado de confianza, es decir, en } \\
\text { principio nadie trata de sacar provecho de la relación, incluso si surge la } \\
\text { oportunidad }\end{array}$ \\
\hline & $\begin{array}{l}\text { 4.2 Considera que su reputación afecta a las organizaciones con las que tiene } \\
\text { relaciones (centros educativos, proveedores) y a su vez la de ellos afecta a su } \\
\text { centro (por ejemplo, si otro centro incumple con las familias o alumnado esto } \\
\text { le repercute a su centro) }\end{array}$ \\
\hline & $\begin{array}{l}4.3 \text { Cree que las relaciones de cooperación que su centro tiene en la actualidad } \\
\text { (proveedores, instituciones, etc.) se mantendrán en el futuro (reciprocidad). Es } \\
\text { decir, cuando ustedes realizan un favor, ¿creen que será compensado en el } \\
\text { futuro? }\end{array}$ \\
\hline
\end{tabular}

Tabla 1. Dimensiones de Redes sociales e ítems

Se ha incorporado al modelo una variable relacionada con el tamaño del centro, haciéndola operativa a partir del número total de alumnos/as del centro. El objetivo de esta inclusión es determinar si el tamaño de la organización tiene incidencia sobre la innovación.

Por otra parte, la variable innovación se hizo operativa a partir de la media de los ítems relacionados con los factores que inciden en la Innovación Educativa de un Centro de Enseñanza descritos en el apartado 3 de este trabajo y que fueron agrupados en los siguientes bloques:

- Innovación sobre objetivos y estructura de la formación. Incluye preguntas sobre el proyecto educativo, curriculum, revisión periódica de objetivos, asignaturas y nuevas actividades.

- Innovación sobre Recursos TIC e Infraestructuras físicas. Ítems sobre la cobertura de Internet, equipación de aulas, uso de dispositivos electrónicos en el aula, biblioteca y otros recursos TIC.

- Innovación sobre aspectos metodológicos. Sistema de evaluación del alumnado, rol del profesor como impulsor, aprendizaje cooperativo, autonomía del alumnado, flexibilidad y adaptación a los cambios.

- Innovación sobre Formación y Apoyo al profesorado. Preguntas relacionadas con el apoyo al profesorado en el uso de nuevas tecnologías o metodologías, formación del profesorado, buenas prácticas compartidas, impulso al profesorado para experimentar en el aula. 
- Innovación sobre la colaboración con las familias. Participación de los padres y madres en la labor del centro, conexión con las familias, organización de actos conjuntos familias-centro.

- Innovación sobre temas organizativos. Existencia de planes de innovación en el centro, certificados de calidad.

- Innovación sobre el impacto de las innovaciones en los últimos tres años respecto a los resultados obtenidos. Ítems relacionados con las mejoras obtenidas, evolución del fracaso escolar en el centro y mejoras competenciales tanto en el profesorado como el alumnado.

Los datos han sido analizados con el programa SPSS, versión 24. La consistencia del cuestionario se ha validado calculando el coeficiente Alfa de Cronbach para cada bloque de preguntas de escala Likert. Su valor oscilará entre 0 y 1 , donde 0 indica ausencia de correlación y valores cercanos a 1 mayor correlación. En nuestro caso, se puede concluir que en general las escalas de medida de las variables analizadas presentan una elevada fiabilidad en su consistencia interna, ya que todas ellas alcanzan un Alfa de Cronbach superior a 0,80 (Tabla 2).

\begin{tabular}{lccc}
\hline & Estadísticas de fiabilidad & \\
\hline $\begin{array}{l}\text { Bloque del } \\
\begin{array}{l}\text { Cuestionario } \\
\text { Analizado }\end{array}\end{array}$ & $\begin{array}{c}\text { Alfa de } \\
\text { Cronbach }\end{array}$ & $\begin{array}{c}\text { Alfa de Cronbach } \\
\text { basada en elementos } \\
\text { estandarizados }\end{array}$ & $\begin{array}{c}\text { N de } \\
\text { elementos }\end{array}$ \\
\hline $\begin{array}{l}\text { Redes de } \\
\text { Capital Social }\end{array}$ &, 804 &, 811 & 11 \\
\hline $\begin{array}{l}\text { Innovación } \\
\text { Educativa }\end{array}$ &, 923 &, 928 & 37 \\
\hline
\end{tabular}

Tabla 2. Validación del cuestionario realizado

Con la información recogida en el cuestionario, en primer lugar, se ha realizado un análisis descriptivo del perfil de los centros educativos de la muestra, obteniendo la información que de forma resumida se indica en la Tabla 3 (la composición de la muestra sigue valores porcentuales, en cuanto al tipo de centro educativo y ubicación, semejantes al total de la población):

\begin{tabular}{|c|c|}
\hline Dato de la Muestra & Valores \\
\hline Fecha de Constitución del Centro & $\begin{array}{l}19,7 \% \text { antes de } 1970,38 \% \text { entre } 1971-95 \text { y } 42,3 \% \text { después de } \\
1996\end{array}$ \\
\hline Tipo de centro Educativo & El $22,5 \%$ son centros privados y el $77,5 \%$ públicos \\
\hline Ubicación (provincia) & $39,4 \%$ en Alicante, $12,7 \%$ en Castellón y $47,9 \%$ en Valencia \\
\hline Número de profesores del centro & El $12,7 \%$ entre 20 y $50,87,3 \%$ más de 50 \\
\hline Número total de alumnado del centro & $\begin{array}{l}\text { El } 11,3 \% \text { entre } 200 \text { y } 400,9,9 \% \text { entre } 400 \text { y } 500,57,7 \% \text { entre } \\
500 \text { y } 1000,21,1 \% \text { más de } 1000\end{array}$ \\
\hline $\begin{array}{l}\text { Número total de alumnado de } \\
\text { Bachillerato }\end{array}$ & $\begin{array}{l}\text { El } 2,8 \% \text { menos de } 30 \text { alumnos, } 14,1 \% \text { entre } 30 \text { y } 60,29,6 \% \\
\text { entre } 60 \text { y } 100,38 \% \text { entre } 100 \text { y } 200 \text {, y el } 15,5 \% \text { más de } 200\end{array}$ \\
\hline
\end{tabular}

Tabla 3. Perfil de los Centros Educativos 


\section{RESULTADOS}

\subsection{Estudio Empírico I}

En esta parte del trabajo se van a contrastar las hipótesis H1 y H2 de nuestro análisis. Estudiamos en qué medida las dimensiones que explican la estructura de las redes de capital social influyen sobre el volumen de innovación en los centros de Bachillerato de la muestra. Planteamos un modelo de regresión lineal múltiple, utilizando como variable dependiente la innovación. Previamente a la aplicación de este modelo de regresión y con el objetivo de evitar posibles correlaciones entre los ítems que conforman las diferentes variables de redes de capital social, hemos llevado a cabo un análisis factorial de componentes principales.

En un análisis factorial es importante contrastar mediante la prueba de esfericidad de Barlett si es o no apropiado someter el modelo al proceso de factorización. Como muestra la Tabla 4 el valor de la prueba resultante es una Chi-cuadrado de 240,957, que con 66 grados de libertad es significativo al, $000(\mathrm{p}<, 01)$, lo que revela que la matriz de correlaciones es significativa.

\begin{tabular}{|c|c|c|}
\hline \multicolumn{3}{|c|}{ Prueba de KMO y Bartlett } \\
\hline \multicolumn{2}{|c|}{ Medida Kaiser-Meyer-Olkin de adecuación de muestreo } & ,750 \\
\hline \multirow{3}{*}{ Prueba de esfericidad de Bartlett } & Aprox. Chi-cuadrado & 240,957 \\
\hline & g1 & 66 \\
\hline & Sig. &, 000 \\
\hline
\end{tabular}

Tabla 4. Prueba de esfericidad de Barlett

Por otra parte, para medir la idoneidad de los datos para realizar un análisis factorial comparando los valores de los coeficientes de correlación observados con los coeficientes de correlación parcial, se ha utilizado la medida de adecuación muestral Kaiser-Meyer-Olkin (KMO) que muestra un valor de 0,750 , muy por encima de 0,5 , que indica un resultado meritorio de adecuación global de la muestra (Kaiser y Rice, 1974).

Finalmente, se ha procedido a realizar el análisis factorial con el método de extracción de los factores de componentes principales, explicando estos más del 63\% de la varianza total. Para facilitar la interpretación de los factores, éstos se han rotado con el criterio varimax, usando la normalización de Kaiser, obteniendo un total de 4 factores, que por la naturaleza de los ítems responden a las dimensiones de análisis descritas en el punto 2 de este trabajo. Con esta información, trabajaremos en la regresión lineal múltiple como sustitución de las medias muestrales de las variables originales (Tabla 5).

Por tanto, conformaremos un modelo de regresión lineal múltiple donde la variable dependiente será la Innovación y las variables independientes: la Dimensión Estructural (DIMestructural), Dimensión Relacional-Riqueza (DIMrelacRiquez), Dimensión Relacional-Fortaleza (DIMrelacFortalez) y Dimensión Normas y Valores (DIMnormValores). Incluiremos como variable de control el tamaño del centro educativo (TAMAÑO CENTRO). 


\begin{tabular}{cccccc}
\hline & Ítems que incluye & \multicolumn{4}{c}{ Componentes } \\
\hline FACTORES & & 1 & 2 & 3 & 4 \\
\hline & 1.3 &, 850 &, 051 &, 091 &, 156 \\
\cline { 2 - 6 } & 2.3 &, 644 &, 319 &,- 121 &, 176 \\
\cline { 2 - 6 } DIMENSIÓN ESTRUCTURAL & 1.1 &, 641 &, 500 &, 059 &, 033 \\
\cline { 2 - 6 } & 2.1 &, 613 &, 016 &, 491 &, 088 \\
\cline { 2 - 6 } & 1.2 &, 586 &,- 197 &, 257 &,- 004 \\
\cline { 2 - 6 } & 3.2 &, 243 &, 793 &,- 016 &, 146 \\
\cline { 2 - 6 } DIMENSIÓN RELACIONAL & 3.1 &,- 075 &, 729 &, 151 &,- 075 \\
(RIQUEZA) & 3.3 &, 159 &, 618 &, 206 &, 520 \\
\hline & 4.3 &,- 017 &, 429 &, 377 &, 199 \\
\hline DIMENSIÓN RELACIONAL & 4.1 &, 146 &, 148 &, 862 &, 142 \\
\hline (FORTALEZA) & 2.2 &, 425 &, 374 &, 437 &,- 277 \\
\hline DIMENSIÓN NORMAS Y VALORES & 4.2 &, 166 &, 075 &, 111 &, 888 \\
\hline
\end{tabular}

Tabla 5. Matriz de factores rotados del análisis de componentes principales de las dimensiones de Redes de Capital Social

En la Tabla 6, podemos observar cómo la bondad de ajuste tiene un valor de 0,163 , lo que implica un porcentaje explicativo de la innovación relativamente aceptable. Por otro lado, el estadístico $\mathrm{F}$ nos muestra que el modelo es significativo y que hay evidencia de que al menos una variable independiente afecta a la Innovación.

\begin{tabular}{ccccccc}
\hline Modelo & $\mathrm{R}$ & $\begin{array}{c}\mathrm{R} \\
\text { cuadrado }\end{array}$ & $\begin{array}{c}\text { R cuadrado } \\
\text { ajustado }\end{array}$ & $\begin{array}{c}\text { Cambio en } \mathrm{R} \\
\text { cuadrado }\end{array}$ & $\begin{array}{c}\text { Cambio en } \\
\mathrm{F}\end{array}$ & $\begin{array}{c}\text { Sig. C } \mathrm{C}^{\mathrm{o}} \text { en } \\
\mathrm{F}\end{array}$ \\
\hline $\mathbf{1}$ &, $485 \mathrm{a}$ &, 235 &, 163 &, 235 & 3,275 &, 007 \\
\hline
\end{tabular}

Tabla 6. Bondad del ajuste del modelo propuesto

En relación con los coeficientes de regresión relacionados con las Dimensiones de las Redes de Capital Social, podemos observar en la Tabla 7 que la "Dimensión Estructural", "Dimensión Relaciones-Riqueza" y "Dimensión Relaciones-Fortaleza" al margen de no ser significativas en el modelo, su relación positiva con la Innovación es muy pequeña y que la única dimensión que afecta a la innovación en los centros de Bachillerato analizados es la dimensión "Normas y Valores"; por tanto, rechazaríamos parcialmente la hipótesis $\mathrm{H} 1$.

Finalmente, podemos destacar que el tamaño del centro no influye en su grado de Innovación por lo que rechazaríamos la Hipótesis $\mathrm{H} 2$.

\subsection{Estudio Empírico II}

Esta parte del estudio hace referencia a las hipótesis $\mathrm{H} 3$ y H4, que contrastan en qué medida hay diferencias significativas en las dimensiones de las Redes Sociales de capital entre los centros públicos y privados de la muestra y si el grado de Innovación difiere en ambos tipos de centros. Para ello, se ha realizado la prueba t de Student para la igualdad de medias para muestras independientes. 


\begin{tabular}{|c|c|c|c|}
\hline \multirow{2}{*}{ Modelo } & \multicolumn{2}{|c|}{ Coeficientes no estandarizados } & \multirow{2}{*}{ Sig. } \\
\hline & B & Error estándar & \\
\hline (Constante) & 1,233 &, 575 & ,036 \\
\hline TAMAÑO DEL CENTRO & ,008 & ,068 & ,908 \\
\hline DIMestructural &, 088 &, 101 & ,390 \\
\hline DIMrelacRiquez & 087 &, 119 & ,468 \\
\hline DIMrelacFortalez &, 066 & ,094 & ,486 \\
\hline DIMnormValores & ,197 & ,078 &, $014^{*}$ \\
\hline
\end{tabular}

Tabla 7. Coeficientes de Regresión y significatividad

\begin{tabular}{lcccc}
\hline \multirow{2}{*}{ Dimensión de Estudio } & Tipo de Centro & $\mathrm{N}$ & Media & $\begin{array}{c}\text { Desviación } \\
\text { estándar }\end{array}$ \\
\hline DIMestructural & Público & 55 & 3,865 &, 6544 \\
\cline { 2 - 5 } DIMrelacRiquez & Privado & 16 & 3,675 &, 7793 \\
\cline { 2 - 5 } & Público & 55 & 3,968 &, 5774 \\
\hline DIMrelacFortalez & Privado & 16 & 3,921 &, 5378 \\
\cline { 2 - 5 } & Público & 55 & 3,773 &, 60792 \\
\hline DIMnormValores & Privado & 16 & 3,344 &, 8360 \\
\cline { 2 - 5 } & Público & 55 & 3,930 &, 6800 \\
\hline
\end{tabular}

Tabla 8. Contraste medias para las dimensiones de Redes de Capital Social

Como se puede observar en la Tabla 8, existen diferencias en las medias entre las dimensiones analizadas para los centros públicos y privados; sin embargo, es necesario comprobar si estas medias son significativas o no para poder realizar afirmaciones sobre el tema.

\begin{tabular}{lccccc}
\hline \multicolumn{1}{c}{ Dimensiones de Estudio } & \multicolumn{2}{c}{$\begin{array}{c}\text { Prueba de Levene } \\
\text { de Igualdad de } \\
\text { Varianzas }\end{array}$} & \multicolumn{2}{c}{ Análisis del estadístico t } \\
\hline DIMestructural & $\begin{array}{c}\text { Se asumen varianzas } \\
\text { iguales }\end{array}$ & 1,390 &, 24 &, 98 & F \\
\hline DIMrelacRiquez & $\begin{array}{c}\text { Se asumen varianzas } \\
\text { iguales }\end{array}$ &, 0040 &, 95 &, 28 &, 77 \\
\hline DIMrelacFortalez & $\begin{array}{c}\text { Se asumen varianzas } \\
\text { iguales }\end{array}$ & 1,762 &, 18 & 2,05 &, 04 \\
\hline DIMnormValores & $\begin{array}{c}\text { Se asumen varianzas } \\
\text { iguales }\end{array}$ & 1,477 &, 22 &,- 59 &, 55 \\
\hline
\end{tabular}

Tabla 9. Significatividad de las medias de las dimensiones de las Redes de Capital Social 
La prueba de Levene para la igualdad de varianzas indica si se puede o no suponer varianzas iguales. Así, como se puede observar en la Tabla 9, si la probabilidad asociada al estadístico de Levene es $>, 05$ se suponen varianzas iguales, si es $<, 05$ varianzas distintas. En la misma tabla, del conjunto de variables relacionadas con las dimensiones de las Redes, todas las dimensiones asumen varianzas iguales, con probabilidad de equivocarse al rechazar esta hipótesis de un mínimo de 0,189 , superior al límite de 0,05. Después de esta observación, se analiza el estadístico t con su nivel de significación bilateral, este valor nos informa sobre el grado de compatibilidad entre la hipótesis de igualdad de medias y las diferencias entre medias poblacionales observadas. En nuestro caso solo la Dimensión Relacional-Fortaleza es significativa $(\mathrm{p}<, 1)$; por lo que es la única dimensión donde se encuentran diferencias de medias significativas entre los centros públicos y privados, por tanto, rechazamos parcialmente la Hipótesis H3, concluyendo que las redes sociales de los centros educativos públicos y privados solo difieren en su Dimensión Relacional-Fortaleza.

Por lo que respecta a la Innovación, al aplicar la prueba de Kolmogorov-Smirnov para las dos muestras (centros públicos y privados), se puede comprobar que ambas muestras siguen una distribución Normal. La muestra correspondiente a los centros privados tiene una puntuación media de 3,54 y una desviación estándar de 0,53 en Innovación, con un p>,05 (Tablas 10 y 11).

\begin{tabular}{ccccc}
\hline Variable de Estudio & Tipo de Centro & $\mathrm{N}$ & Media & $\begin{array}{c}\text { Desviación } \\
\text { estándar }\end{array}$ \\
\hline \multirow{2}{*}{ INNOVACIÓN } & Público & 55 & 3,265 &, 5189 \\
\cline { 2 - 5 } & Privado & 16 & 3,543 &, 5344 \\
\hline
\end{tabular}

Tabla 10. Contraste medias para la variable Innovación

En los centros públicos el valor de la media se sitúa en un nivel ligeramente inferior con 3,26 puntos y desviación estándar de 0,52 , con $\mathrm{p}>, 05$. En la Tabla 11 podemos ver que asumiendo varianzas iguales esta diferencia de medias es significativa tomando un valor de $\mathrm{p}<, 1$, que es razonable para este tipo de análisis, por lo que se podría afirmar que hay diferencia entre el grado de Innovación presente en los centros públicos y privados, con un valor ligeramente superior en estos últimos, aceptando la hipótesis H4.

\begin{tabular}{lccccc}
\hline Variable de Estudio & \multicolumn{2}{c}{$\begin{array}{c}\text { Prueba de Levene de } \\
\text { Igualdad de Varianzas }\end{array}$} & \multicolumn{2}{c}{ Análisis del estadístico t } \\
\hline & F & Sig. & t & Sig.(bilateral) \\
\hline INNOVACIÓN & Se asumen varianzas iguales &, 095 &, 759 & $-1,877$ &, 065 \\
\hline
\end{tabular}

Tabla 11. Significatividad de las medidas para la variable Innovación

\section{CONCLUSIONES}

El uso de las redes de capital social como medio para incrementar la eficacia y la eficiencia de una organización no es un tema novedoso y ha sido estudiado en diferentes tipos de industrias (Powell, 1987; Lorenzoni y Ornati, 1988; Jarillo, 1988). Sin embargo, pese a la relevancia que puede llegar a tener en el área educativa, no hay 
muchos estudios que hayan profundizado en los efectos y las ventajas de pertenecer a una red social. Sabemos por experiencia que los centros de enseñanza son organizaciones personalistas, donde el conocimiento suele descansar en la base de la organización (profesorado). Este aspecto, que por una parte supone una gran ventaja frente a otro tipo de organizaciones, dado que desaparecen los filtros intraorganizacionales, también puede suponer una individualización del conocimiento y un aislamiento que impida generar capital social entre los centros.

Las redes proporcionan poder, información, conocimiento y capital, facilitando la capacidad de aprendizaje y la habilidad para innovar. Pero, en este contexto es importante tener en cuenta que las redes están formadas por dos componentes: el interpersonal (contactos entre profesorado en nuestro caso) y el interorganizacional (contactos a nivel de centro educativo) y, como se ha visto en este trabajo, la mejora de la organización se consigue a través de las redes interorganizacionales, aunque las interpersonales son necesarias para mantener activas éstas. Por otra parte, se genera capital social cuando hay confianza entre los actores que intervienen en la red, por lo que si unimos todos estos aspectos podemos observar que la figura del profesorado constituye la base y quizás el todo para que las redes sean efectivas y se traduzcan en innovación.

La innovación es un concepto muy amplio, aunque lo podemos centrar sobre la producción y difusión de prácticas para producir mejoras. Para el estudio de la innovación educativa es importante que tengamos en cuenta que ésta se origina tanto en el aula como en el centro escolar, la administración educativa y la propia comunidad. Esto la hace más compleja en su análisis, teniendo en cuenta que un centro adquiere la reputación de innovador a partir de la participación de los múltiples actores que tienen influencia sobre éste. Por otra parte, la estructura normativa a la que están sujetos los centros provoca que la innovación muchas veces dependa más de la voluntad de profesoras y profesores que de las propias políticas que puedan implementar tanto la Administración pública como los centros educativos. No estamos diciendo con ello que estas políticas no sean necesarias, más bien al contrario, sino que para su implementación es necesario que el profesorado disponga de niveles adecuados de incentivos, motivación y recursos a su disposición.

Solo seremos capaces de innovar en un centro de enseñanza si éste dispone de los recursos materiales adecuados (infraestructura, TICs, etc.) y humanos (formación del profesorado, equipo directivo, soporte del personal no docente, alumnado predispuesto a generar e implementar ideas y familias que participen en el proceso formativo), además de un entorno que valore el esfuerzo de los profesionales de la enseñanza e incentive su contribución. Todo ello en el marco de políticas orientadas al conocimiento, la creatividad y la innovación formalizadas en Proyectos de Centro.

En la Comunidad Valenciana hay un total de 471 centros homologados de Bachillerato registrados por la Consellería d'Educació, Investigació, Cultura i Esport, de los que el $70 \%$ son públicos y el resto privados (tanto concertados como no). Una de las primeras cuestiones que puede resultar interesante en su análisis es comprobar si el comportamiento de ambos tipos de centro es igual o difiere en cuanto a las redes de contactos en las que participan y actúan. En este sentido, se ha comprobado que las conductas en ambas muestras (públicos/privados) son semejantes y solo difieren en la Dimensión Relacional-Fortaleza, es decir, los centros públicos dedican más tiempo a 
mantener relaciones con otros centros que los privados, además los contactos son más frecuentes y con mayor intensidad.

Por lo que respecta a la innovación, interesa conocer si el grado de innovación sigue las mismas pautas o por el contrario podemos encontrar diferencias significativas en ambos colectivos. En este sentido, uno de los primeros hallazgos es la homogeneidad entre centros, tal como se desprende del coeficiente de variación de Pearson de ambos, que se sitúa en 0,15 para públicos y 0,14 para privados. Este dato es importante puesto que cuanto más próximo a cero, menor es la dispersión relativa de la muestra y mayor es la representatividad de la media. Por tanto, es interesante observar que no hay disparidad en las actuaciones entre los diferentes centros de enseñanza de Bachillerato, más bien parecen existir pautas de comportamiento muy semejantes en cuanto a innovación a juzgar por los resultados obtenidos. Estos resultados muestran que las diferencias en cuanto al grado de innovación entre los centros públicos y privados es significativa, aunque pequeña, decantándose ligeramente a favor de los privados.

Otro de los retos que plantea este trabajo es conocer si las diferentes Dimensiones que definen una Red de Capital Social identificadas en este estudio condicionan el grado de innovación en los centros educativos. Contrariamente a lo que podríamos pensar, en función de los diferentes estudios que encontramos en la literatura sobre redes e innovación relacionados con otro tipo de organizaciones, en los centros educativos de Bachillerato solo la Dimensión "Normas y Valores" tiene un impacto sobre el grado de innovación educativa en el centro. Estamos diciendo que la estructura de la Red, la cantidad de tiempo que se dedica a las relaciones de contacto, la intensidad de estos contactos y su frecuencia no se vuelcan sobre la innovación de los centros de enseñanza directamente.

También es interesante comprobar que los centros no perciben, en la mayor parte de los contactos, un intercambio de información valiosa entre ellos (por lo que se desprende de la baja significatividad de la Dimensión Relacional-Riqueza). Sin embargo, sí que le otorgan peso a la Dimensión "Normas y Valores". Estamos hablando de una variable que hace referencia a la reputación del centro, la confianza y la penalización por comportamientos oportunistas; por lo que podemos pensar que se intercambia información adecuada y de calidad cuando hay un grado suficiente de confianza entre los partícipes, guiados por la propia reputación tanto del profesorado como del centro. En este sentido, sabemos que la reputación se consigue actuando, por lo que aquellos centros más innovadores generan una reputación que abre un bucle para la cooperación con otros y por lo tanto su crecimiento y mejora en materia educativa.

Finalmente, es importante comprobar si el tamaño del centro tiene incidencia directa sobre su grado de Innovación como ocurre en gran parte de las organizaciones empresariales. De nuestro estudio se puede observar que, al margen de no ser significativa la variable, el impacto positivo es muy pequeño sobre la innovación; por lo que podemos afirmar que el tamaño no incide en la capacidad de innovar de un centro de enseñanza de Bachillerato.

Como toda investigación, este trabajo tiene una serie de limitaciones. En primer lugar, hemos partido de las dimensiones sobre las Redes de Capital Social identificadas en la literatura especializada sobre el tema. Podría ocurrir que, dada la particularidad de este tipo de organizaciones, existieran otras variables adicionales de Redes de Capital Social por las que no se ha preguntado y que tuvieran alguna incidencia sobre el grado 
de innovación del Centro. Por otra parte, hemos analizado la innovación como una variable única tratándola como una media; sin embargo, ha sido conformada por la combinación de diferentes dimensiones que no han sido consideradas de forma independiente en este trabajo y que nos podrían indicar semejanzas y diferencias parciales en cuanto al grado de innovación de los centros.

Este trabajo pretende tener una continuidad de forma inmediata. En primer lugar, se analizarán los resultados obtenidos en las diferentes dimensiones de la innovación identificadas en este estudio, conociendo cuáles son los puntos fuertes y las carencias que los centros indican en cuanto a su capacidad de innovar en materia educativa y si existen diferencias entre los centros públicos y privados sobre los diferentes componentes de la innovación. También hemos considerado interesante profundizar, a partir de una investigación cualitativa, en el grado de conocimiento del por qué se está produciendo la situación que estamos mostrando, máxime teniendo en cuenta que existe la creencia de que en Bachillerato no se utilizan métodos más innovadores porque la formación se orienta a que el alumnado consiga resultados exitosos en la prueba de acceso a la Universidad (Luzarraga, Núñez y Etxeberría, 2018).

Finalmente, entendemos que este estudio puede ser reproducible en otras Comunidades Autónomas, proporcionando información sobre los diferentes comportamientos de los centros de enseñanza y aportando indicios que permitan a las Comunidades intercambiar prácticas orientadas a la mejora de la innovación educativa.

\section{BIBLIOGRAFÍA}

Almuiñas, J.L. y Galarza, J. (2016). Las redes académicas como ejes de integración y cooperación internacional de las instituciones de educación superior. Revista Cubana de Educación Superior, 35(1), 18-29.

Azqueta, D., Gavaldón, G. y Margalef, L. (2007). Educación y desarrollo: ¿capital humano o capital social? Revista de educación, 344, 265-283.

Burt, R.S.(1992). Structural holes. Cambridge. MA: Harvard University Press.

Cabaleiro, M.J. (2001). Redes de empresas: propuesta de crecimiento externo para las sociedades cooperativas. REVESCO. Revista de Estudios Cooperativos, 74, 7-22.

Carolan, B.V. (2013). Social network analysis and education. Theory, methods, and applications. Thousand Oaks, CA: Sage Publications.

Casillas, S., Cabezas, M. y Navarro, L. (2013): Innovación Educativa en los Centros de Enseñanza. Revista Galego-Portuguesa de Psicoloxía e Educación, 21, 137-148.

Casson, M. y Giusta, M.D. (2007). Entrepreneurship and social capital: Analysing the impact of social networks on entrepreneurial activity from a rational action perspective. International small business journal, 25(3), 220-244.

Chow, W.S. y Chan, L.S. (2008). Social network, social trust and shared goals in organizational knowledge sharing. Information \& management, 45(7), 458-465.

Colás, M.P., De Pablos, J. y Ballesta, J. (2018). Incidencia de las TIC en la enseñanza en el sistema educativo español: una revisión de la investigación. Revista de Educación a Distancia (RED), 18(56). Recuperado de: https://revistas.um.es/red/article/view/321471

Elfring, T. y Hulsink, W. (2003). Networks in entrepreneurship: The case of high-technology firms. Small business economics, 21(4), 409-422.

García-Valcárcel, A., Hernández, A. y Recamán, A. (2012). La metodología del aprendizaje colaborativo a través de las TIC: una aproximación a las opiniones de profesores y alumnos. Revista complutense de educación, 23(1), 161-188.

González, A. y De Pablos, J. (2015). Factores que dificultan la integración de las TIC en las 
aulas. Revista de Investigación Educativa, 33(2), 401-417.

Granovetter, M.S. (1973). The Strength of weak ties. American Journal of Sociology, 78, 1.3601.380 .

Herrera, H. (2009). Investigación sobre redes sociales y emprendimiento: revisión de la literatura y agenda futura. Innovar, 33, 19-33.

Hung, H. (2006). Formation and survival of new ventures: A path from interpersonal to interorganizational networks. International Small Business Journal, 24(4), 359-378.

Jarillo, J.C. (1988). On strategic networks. Strategic management journal, 9(1), 31-41.

Kaiser, H. y Rice, J. (1974). Little jiffy, mark IV. Educational and psychological measurement, 34(1), 111-117.

Lin, N. (2001). Social capital: A theory of social structure and action (1ST Ed.) New York: Cambridge University Press.

Llorens, F. (2011). Identidad Digital e Innovación en los Centros Educativos. En J. Hernández Ortega, Massimo Pennesi. D. Sobrino López y Azucena Vázquez Gutiérrez (coords.), Experiencias educativas en las aulas del siglo XXI (pp. 315-321). Barcelona: Ariel.

López, S., Civís, M. y Díaz-Gibson, J. (2018). Capital Social y Redes Sociales de Maestros: Revisión Sistemática. Revista de Educación, 381, 233-257.

Lorenzoni, G. y Ornati, O. (1988). Constellations of firms and new ventures. Journal of Business venturing, 3(1), 41-57.

Luzarraga Martín, J.M., Núñez Lozano, J.M. y Etxeberría Murgiondo, J. (2018). Análisis de las expectativas de los centros de Bachillerato de alta y baja eficacia escolar. Percepción de la Inspección Educativa. Revista Complutense de Educación, 29(4), 1.075-1.090.

Marquès, P. (2005). Cambios en los centros educativos: construyendo la escuela del futuro. Didáctica, Innovación y Multimedia, 1-39. Disponible en: https://www.raco.cat/index.php/DIM/article/view/56081/65503

Molina Morales, F.X. (2005). The territorial agglomerations of firms: A social capital perspective from the Spanish tile industry. Growth and Change, 36(1), 74-99.

Muñoz-Fernández, G.A., Rodríguez-Gutiérrez, P. y Luque-Vílchez, M. (2019). La formación inicial del profesorado de educación secundaria en España: perfil y motivaciones del futuro docente. Educación XX1, 22(1), 71-92.

Parra, S., Gómez, M. y Pintor, M. (2015). Factores que inciden en la implementación de las TIC en los procesos de enseñanza-aprendizaje en $5^{\circ}$ de Primaria en Colombia. Revista Complutense de educación, 26, ( $\mathrm{n}^{\circ}$ Especial: TIC en Educación), 197-213.

Pericacho, J. (2014). Pasado y presente de la renovación pedagógica en España (de finales del Siglo XIX a nuestros días). Un recorrido a través de escuelas emblemáticas. Revista Complutense de Educación, 25(1), 47-67.

Powell, W.W. (1987). Hybrid organizational arrangements: new form or transitional development? California management review, 30(1), 67-87.

Rosales, C. (2012). Contextos de la Innovación Educativa. Innovación Educativa, 22, 9-21.

Tsai, W. y Ghoshal, S. (1998). Social capital and value creation: The role of intrafirm networks. Academy of management Journal, 41(4), 464-476.

Venkataraman, S. y Van de Ven, A. (1998). Hostile environmental jolts, transaction set, and new business. Journal of Business Venturing, 13(3), 231-255. 